\title{
Emphysematous Cholecystitis Secondary to Fusobacterium nucleatum
}

\author{
Anuj Kunadia ${ }^{1}$, Michael B. Leong ${ }^{2}$, Karthikram Komanduri ${ }^{3,4}$, Randa Abdelmasih ${ }^{3}$, Aneta Tarasiuk- \\ Rusek $^{5}$ \\ 1. Internal Medicine, University of Central Florida College of Medicine, Orlando, USA 2. Internal Medicine, University \\ of Central Florida, Orlando, USA 3. Internal Medicine, Ocala Regional Medical Center, University of Central Florida \\ College of Medicine, Ocala, USA 4. Internal Medicine, Ocala Regional Medical Center, University of Central Florida, \\ Ocala, USA 5. Infectious Disease, Ocala Regional Medical Center, University of Central Florida College of Medicine, \\ Ocala, USA
}

Corresponding author: Anuj Kunadia, anujkunadia@knights.ucf.edu

\begin{abstract}
Fusobacterium nucleatum may be implicated in cases of emphysematous cholecystitis (EC) and carries a high mortality risk, especially in individuals with heart disease, renal insufficiency, and underlying malignancy. Fusobacterium infections are rarely detected in the setting of cholecystitis possibly due to the difficulty with properly culturing the bacteria. We describe a case of a patient with EC in whom blood cultures were positive for growth of $F$. nucleatum in one of two samples. The patient was treated with empiric antibiotic therapy consisting of metronidazole and cefepime. In patients with EC and negative cultures, it is possible that they may have an undetected infection with fusobacteria, which carries a high mortality risk. As such, clinicians should maintain a high degree of suspicion of obligate anaerobic infection in patients who have negative blood culture for growth in the setting of EC and consider continuation of adequate antimicrobial coverage.
\end{abstract}

Review began 05/17/2021 Review ended 06/06/2021 Published 06/15/2021

(c) Copyright 2021 Kunadia et al. This is an open access article distributed under the terms of the Creative Commons Attribution License CC-BY 4.0., which permits unrestricted use, distribution, and reproduction in any medium, provided the original author and source are credited.
Categories: Internal Medicine, Infectious Disease

Keywords: emphysematous cholecystitis, fusobacterium nucleatum emphysematous cholecystitis, culture negative, anaerobic, fusobacterium nucelatum

\section{Introduction}

Emphysematous cholecystitis (EC) is an acute variant of cholecystitis, characterized by air in the gallbladder lumen, wall, or surrounding tissues without an abnormal communication with the gastrointestinal tract [1]. Acute cholecystitis is associated with inflammation of the gallbladder, with at least $90 \%$ of cases arising from cholelithiasis. The rare emphysematous variant is associated with less than $3 \%$ of cholecystitis cases with a mortality rate of $15 \%-20 \%$ in comparison to the $1.4 \%$ mortality in patients with cholecystitis. It should be noted that patients with EC may also have concurrent gallstones [2,3]. The underlying etiology is thought to be infection resulting in gangrene and subsequent necrosis of the gallbladder. EC is most commonly seen in males over 50 years old. Other associated medical conditions include cardiovascular disease, diabetes, and neoplasms [4].

Patients usually present with right upper quadrant pain, fever, nausea, vomiting, and occasionally positive Murphy's sign [5,6]. Ultrasound (US) is the best initial imaging modality for detection, although the ability to visualize EC varies based on the amount of air in the gallbladder. The most sensitive imaging technique is CT. Diagnosis of EC is based on the clinical presentation and imaging [5,7]. EC is approached as a lifethreatening condition, so patients should be administered antibiotics and undergo a cholecystectomy as soon as possible.

Secondary bacterial infections in acute cholecystitis are commonly due to the enteric organisms Escherichia coli, Klebsiella, Streptococcus faecalis, and Enterococci [8,9]. Clostridium perfringens is uniquely seen in patients with EC [10].

Our patient was found to have bacteremia secondary to Fusobacterium nucleatum in the setting of EC. Fusobacteria are obligate anaerobic bacteria commonly found in the oropharyngeal and gastrointestinal tracts $[11,12]$. Infection is usually associated with colorectal carcinoma and immunocompromised individuals [13]. Fusobacteria are usually sensitive, with low rates of resistance, to penicillins, metronidazole, and clindamycin $[12,14]$.

\section{Case Presentation}

A 63-year-old male with a history of extensive alcohol and drug use, pancreatitis, hypertension, and gout was brought to the emergency department by emergency medical services due to a few days of stupor. Physical examination was significant for blood pressure of $167 / 87$ and pain in the right upper quadrant. Labs demonstrated anion gap metabolic acidosis, rhabdomyolysis, hyponatremia, elevated liver enzymes, and acute kidney injury with severe uremia. Non-contrast CT of the abdomen and pelvis showed a distended 


\section{Cureus}

gallbladder with an air-fluid level, confirming the diagnosis of EC (Figure 1). These findings were consistent with those found in the initial US (Figure 2). The patient received an emergency percutaneous

cholecystostomy tube (Figure 3) and was started on empiric antibiotic therapy consisting of metronidazole and cefepime. Later in the course of admission, one of two blood cultures was positive for growth of F. nucleatum.

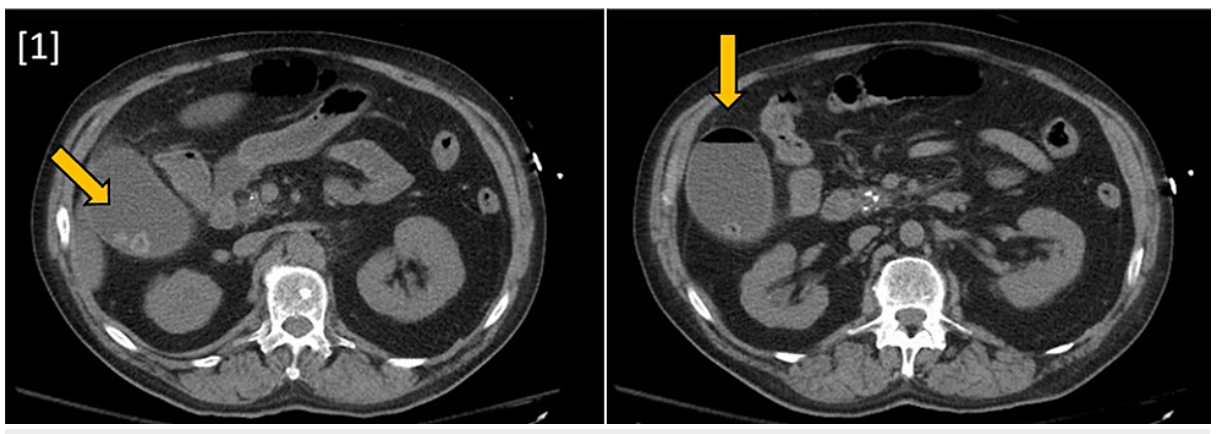

FIGURE 1: CT abdomen and pelvis without contrast showing a distended gallbladder with air-fluid collection and multiple stones as well as a peri-cholecystic fluid reaction.
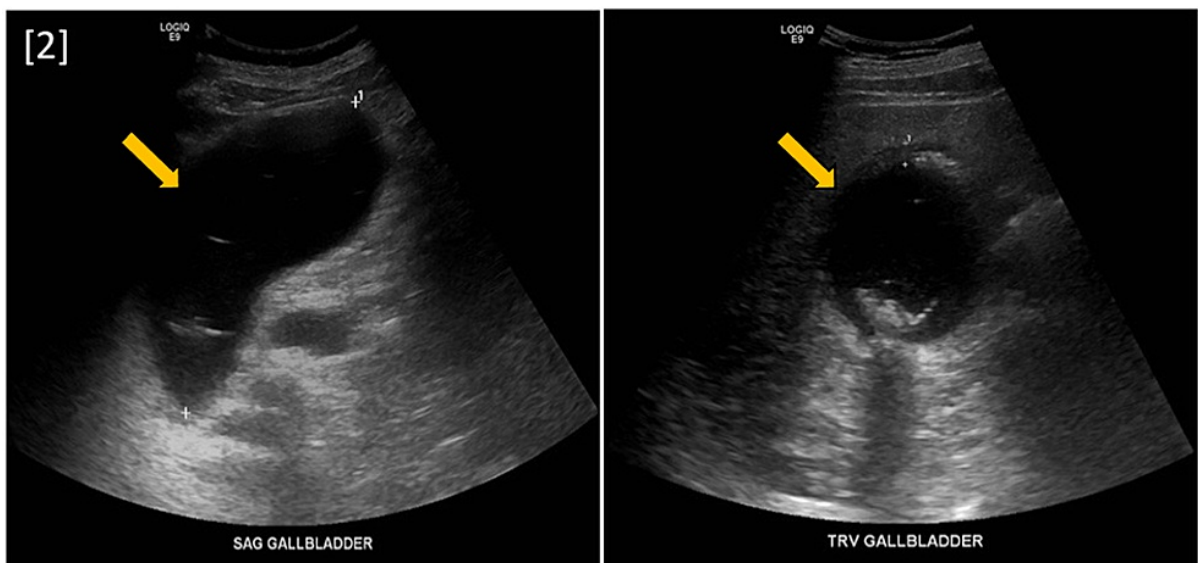

FIGURE 2: Right upper quadrant ultrasound (sagittal and transverse sections) showing moderately distended gallbladder with sludge and stones. Additionally, air present in the fundus is highly suggestive of emphysematous cholecystitis.
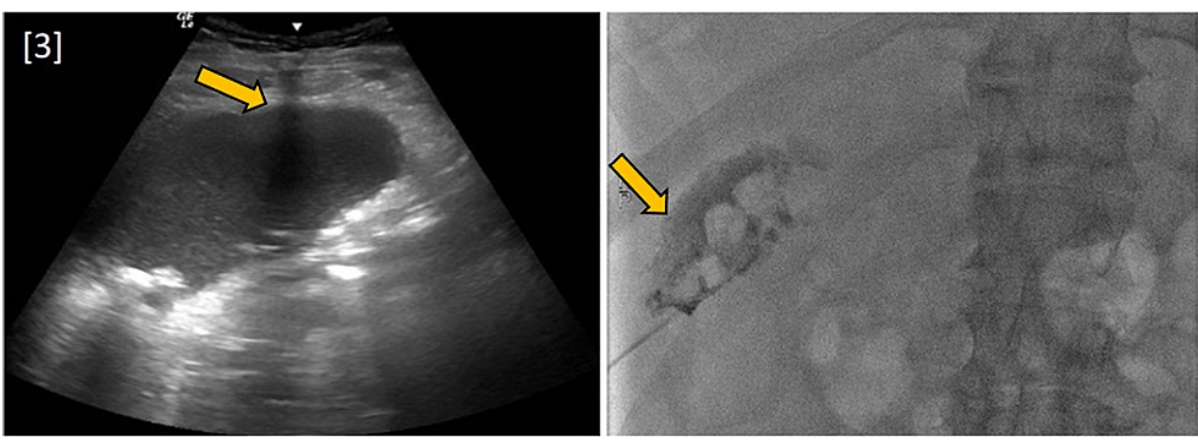

FIGURE 3: Fluoroscopic image-guided percutaneous cholecystostomy tube placement.

Discussion 
The prevalence of Fusobacterium in cholecystitis is likely underestimated due to the low incidence of positive blood cultures and the methods of obtaining fluid samples. Fusobacterium nucleatum is a known cause of odontogenic infections, pleural empyemas, and brain abscesses [15]. Fusobacterium nucleatum infections can also precipitate Lemierre Syndrome, a thrombophlebitis of the internal jugular vein [16]. However, Fusobacterium bacteremia is an uncommon infection, which accounts for approximately $0.9 \%$ of patients with bacteremia and carries an overall mortality rate of $40.7 \%$ even when patients receive appropriate antibiotic treatment. Heart failure, renal insufficiency, and malignancy are independent risk factors for mortality [17]. The low prevalence of Fusobacterium blood cultures is partially due to the fact that the organism is an obligate anaerobe. One study showed that appendicitis caused by anaerobic organisms only resulted in a positive blood culture $50 \%$ of the time [18].

Another source of low prevalence of EC secondary to Fusobacterium may be the method through which culture specimens are obtained. The specimens must be obtained and transported in an anaerobic environment. There are multiple documented cases of patients infected with F. nucleatum in which specimen cultures were negative for growth. In these cases, the infection was only detected when 16S rRNA gene polymerase chain reaction of the sample was utilized [19].

Cholecystostomy tubes are an easy way to access fluid from the gallbladder, especially in patients who are not candidates for cholecystectomy. However, the tube exposes the fluid to air, which can kill obligate anaerobic bacteria. Directly collecting sample fluid in an anaerobic environment from the gallbladder may be more likely to grow the bacteria responsible for EC. Identification of Fusobacterium may not alter management in the setting of acute cholecystitis as it is sensitive to current recommended antibiotic therapy [20]. However, clinicians should have suspicion for anaerobic etiology for EC in the setting of negative fluid and blood cultures, and continuation of antibiotic treatment should be considered to cover for Fusobacterium and other obligate anaerobic microorganisms that may be difficult to culture.

\section{Conclusions}

Fusobacteria may be implicated in cases of EC. Bacteremia with this organism carries a high mortality risk, especially in individuals with heart disease, renal insufficiency, and underlying malignancy. Fusobacterium infections are rarely detected in the setting of cholecystitis and this may be due to the difficulty culturing the bacteria with current techniques. Despite negative cultures, patients with EC may have infection with fusobacteria, which carries a high mortality risk. As such, clinicians should maintain a high degree of suspicion of obligate anaerobic infection in patients who have negative blood culture for growth in the setting of EC and consider continuation of adequate antimicrobial coverage.

\section{Additional Information}

\section{Disclosures}

Human subjects: Consent was obtained or waived by all participants in this study. Conflicts of interest: In compliance with the ICMJE uniform disclosure form, all authors declare the following: Payment/services info: All authors have declared that no financial support was received from any organization for the submitted work. Financial relationships: All authors have declared that they have no financial relationships at present or within the previous three years with any organizations that might have an interest in the submitted work. Other relationships: All authors have declared that there are no other relationships or activities that could appear to have influenced the submitted work.

\section{References}

1. Mentzer RM, Jr., Golden GT, Chandler JG, Horsley JS, 3rd: A comparative appraisal of emphysematous cholecystitis. Am J Surg. 1975, 129:10-15. 10.1016/0002-9610(75)90159-2

2. Kowalski A, Kashyap S, Mathew G, Pfeifer C: Clostridial Cholecystitis. StatPearls [Internet]. StatPearls Publishing, Treasure Island, FL; 2021.

3. Mora-Guzmán I, Martín-Pérez E: Perforated emphysematous cholecystitis and Streptococcus bovis . Rev Esp Enferm Dig. 2019, 111:166-167. 10.17235/reed.2018.5826/2018

4. Strasberg SM: Clinical practice. Acute calculous cholecystitis. N Engl J Med. 2008, 358:2804-2811. 10.1056/NEJMcp0800929

5. Safwan M, Penny SM: Emphysematous cholecystitis: a deadly twist to a common disease . J Diagn Med Sonogr. 2016, 32:131-137. 10.1177/8756479316631535

6. Mhamdi S, Mhamdi K: Emphysematous cholecystitis. N Engl J Med. 2019, 381:e14. 10.1056/NEJMicm1814551

7. Al Hammadi F, Buhumaid R: Point-of-care ultrasound diagnosis of emphysematous cholecystitis. Clin Pract Cases Emerg Med. 2020, 4:107-108. 10.5811/cpcem.2019.11.45337

8. Indar AA, Beckingham IJ: Acute cholecystitis. BMJ. 2002, 325:639-643. 10.1136/bmj.325.7365.639

9. Smith EA, Dillman JR, Elsayes KM, Menias CO, Bude RO: Cross-sectional imaging of acute and chronic gallbladder inflammatory disease. AJR Am J Roentgenol. 2009, 192:188-196. 10.2214/AJR.07.3803

10. Yen WL, Hsu CF, Tsai MJ: Emphysematous cholecystitis. Ci Ji Yi Xue Za Zhi. 2016, 28:37-38. 10.1016/j.tcmj.2015.12.001

11. Denes E, Barraud O: Fusobacterium nucleatum infections: clinical spectrum and bacteriological features of 78 cases. Infection. 2016, 44:475-481. 10.1007/s15010-015-0871-x 


\section{Cureus}

12. Ali SA, Kovatch KJ, Smith J, Bellile EL, Hanks JE, Hoff PT: Implication of Fusobacterium necrophorum in recurrence of peritonsillar abscess. Laryngoscope. 2019, 129:1567-1571. 10.1002/lary.27675

13. King M, Hurley H, Davidson KR, Dempsey EC, Barron MA, Chan ED, Frey A: The link between Fusobacteria and colon cancer: a fulminant example and review of the evidence. Immune Netw. 2020, 20:e30. 10.4110/in.2020.20.e30

14. Riordan T: Human infection with Fusobacterium necrophorum (Necrobacillosis), with a focus on Lemierre's syndrome. Clin Microbiol Rev. 2007, 20:622-659. 10.1128/CMR.00011-07

15. Dyrhovden R, Øvrebø KK, Nordahl MV, Nygaard RM, Ulvestad E, Kommedal Ø: Bacteria and fungi in acute cholecystitis. A prospective study comparing next generation sequencing to culture. J Infect. 2020, 80:16-23. 10.1016/j.jinf.2019.09.015

16. Kuppalli K, Livorsi D, Talati NJ, Osborn M: Lemierre's syndrome due to Fusobacterium necrophorum. Lancet Infect Dis. 2012, $12: 808-815.10 .1016 / \mathrm{s} 1473-3099(12) 70089-0$

17. Su CP, Huang PY, Yang CC, Lee MH: Fusobacterium bacteremia: clinical significance and outcomes . J Microbiol Immunol Infect. 2009, 42:336-342.

18. Jiménez A, Sánchez A, Rey A, Fajardo C: Recovery of aerobic and anaerobic bacteria from patients with acute appendicitis using blood culture bottles. Biomedica. 2019, 39:699-706. 10.7705/biomedica.4774

19. Chakvetadze C, Purcarea A, Pitsch A, Chelly J, Diamantis S: Detection of Fusobacterium nucleatum in culture-negative brain abscess by broad-spectrum bacterial 16S rRNA gene PCR. IDCases. 2017, 8:94-95. 10.1016/j.idcr.2017.04.013

20. Gomi H, Solomkin JS, Schlossberg D, et al.: Tokyo Guidelines 2018: antimicrobial therapy for acute cholangitis and cholecystitis. J Hepatobiliary Pancreat Sci. 2018, 25:3-16. 10.1002/jhbp.518 